\title{
Suicide during Perinatal Period: Epidemiology, Risk Factors, and Clinical Correlates
}

\begin{abstract}
Laura Orsolini ${ }^{1,2,3,4 *}$, Alessandro Valchera ${ }^{2,3}$, Roberta Vecchiotti ${ }^{2,3,4}$, Carmine Tomasetti, ${ }^{3,5,6}$, Felice lasevoli ${ }^{3,6}$, Michele Fornaro ${ }^{3,7}$, Domenico De Berardis ${ }^{3,8,9}$, Giampaolo Perna ${ }^{10,11}$, Maurizio Pompili' ${ }^{12}$ and Cesario Bellantuono ${ }^{13}$
\end{abstract}

\begin{abstract}
${ }^{1}$ School of Life and Medical Sciences, University of Hertfordshire, Hatfield, UK, ${ }^{2}$ Villa San Giuseppe Hospital, Hermanas Hospitalarias, Ascoli Piceno, Italy, ${ }^{3}$ Polyedra Research Group, Teramo, Italy, ${ }^{4}$ Department of Psychiatry and Neuropsychology, University of Maastricht, Maastricht, Netherlands, ${ }^{5}$ Department of Mental Health ASL Teramo, Psychiatric Service of Diagnosis and Treatment, NHS, Hospital "Maria SS dello Splendore", Giulianova, Italy, "aboratory of Molecular and Translational Psychiatry, Department of Neuroscience, Reproductive and Odontostomatological Sciences, University of Naples "Federico II", Napoli, Italy, ${ }^{7}$ New York Psychiatric Institute, Columbia University, New York City, NY, USA, ${ }^{8}$ Department of Mental Health ASL Teramo, Psychiatric Service of Diagnosis and Treatment, NHS, Hospital "G. Mazzini", Teramo, Italy, ${ }^{9}$ Department of Neuroscience and Imaging, University "G. d'Annunzio", Chieti, Italy, ${ }^{10}$ Department of Clinical Neurosciences, Hermanas Hospitalarias, FoRiPsi, Villa San Benedetto Menni, Albese con Cassano, Como, Italy, ${ }^{11}$ Department of Psychiatry and Behavioral Sciences, Leonard Miller School of Medicine, University of Miami, Coral Gables, FL, USA, ${ }^{12}$ Department of Psychiatry, Sant'Andrea Hospital, Rome, Italy, ${ }^{13}$ DEGRA Clinic, Verona-Rimini-Ancona, Italy
\end{abstract}

Edited by:

Mauro Giovanni Carta,

University of Cagliari, Italy

Reviewed by:

Sergio Machado,

Federal University of

Rio de Janeiro, Brazil

Giuseppe Tavormina,

Psychiatric Studies Centre, Italy

${ }^{*}$ Correspondence: Laura Orsolini laura.orsolini@hotmail.it

Specialty section: This article was submitted to Affective Disorders and Psychosomatic Research, a section of the journal Frontiers in Psychiatry

Received: 31 March 2016

Accepted: 25 July 2016

Published: 12 August 2016

Citation:

Orsolini L, Valchera A, Vecchiotti $R$, Tomasetti C, lasevoli F, Fornaro $M$, De Berardis D, Perna G, Pompili M and Bellantuono C (2016) Suicide during Perinatal Period: Epidemiology, Risk Factors, and Clinical Correlates.

Front. Psychiatry 7:138. doi: 10.3389/fpsyt.2016.00138
Perinatal period may pose a great challenge for the clinical management and treatment of psychiatric disorders in women. In fact, several mental illnesses can arise during pregnancy and/or following childbirth. Suicide has been considered a relatively rare event during the perinatal period. However, in some mental disorders (i.e., postpartum depression, bipolar disorder, postpartum psychosis, etc.) have been reported a higher risk of suicidal ideation, suicide attempt, or suicide. Therefore, a complete screening of mothers' mental health should also take into account thoughts of suicide and thoughts about harming infants as well. Clinicians should carefully monitor and early identify related clinical manifestations, potential risk factors, and alarm symptoms related to suicide. The present paper aims at providing a focused review about epidemiological data, risk factors, and an overview about the main clinical correlates associated with the suicidal behavior during the pregnancy and postpartum period. Practical recommendations have been provided as well.

Keywords: suicide, suicidal ideation, suicide attempt, pregnancy, perinatal period, puerperium, postpartum

\section{INTRODUCTION}

Suicide was worldwide ranked as the 14th leading cause of mortality and morbidity, and it is expected to increase by $50 \%$, becoming the 12th leading cause of mortality by year 2030 (1). Suicide represents a major public health problem, with more than 1,000,000 suicides worldwide (2). A preexisting vulnerability, such as a family history of suicide, impulsivity, and previous and/or current psychiatric diagnoses, may be precipitating risk factors for suicidal ideation (SI) and behavior $(3,4)$. Specifically, major depressive disorder (MDD) and other affective disorders may represent 
strong risk factors for suicide (2). Furthermore, SI and a history of personal suicidal behavior are among the most salient shortand long-term risk factors for suicide (2). The National Institute of Mental Health (NIMH) Developing Centers for Intervention and Prevention of Suicide defined the SI as the wish to die, thoughts of killing oneself, and the intent to kill oneself (5). Thoughts of killing oneself are thoughts, beliefs, images, voices, or other cognitions about intentionally ending one's own life (suicide) and may include the intent to act on such thoughts (2). SI or thoughts may be often associated with suicide attempts and completions $(6,7)$.

The identification of SI and behavior, as well as high-risk individuals, requires an adequate suicide screening assessment (2), particularly during the perinatal period. Generally, peripartum (including conception, pregnancy, and postpartum) may be a period of considerable vulnerability to MDD and affective disorders as well as it is frequently associated with the onset and/ or recrudescence of a psychiatric illness $(8,9)$. Overall, approximately $10-15 \%$ of newly delivered women experience a major depressive episode; while around $50 \%$ of women with a previous mood disorder and $70 \%$ with a family history of postpartum psychosis will develop a relapse and/recrudescence following a subsequent delivery (10).

Although suicides and suicidal attempts occur at a lower rate during pregnancy and the postpartum period than in general population (10), the prevalence of SI or thoughts ranges from 5 to $14 \%(9,11,12)$ and, sometimes, it may result in a suicide attempts and completions $(6,11)$. In fact, perinatal suicidality, which comprises completed suicides, suicide attempts, SI, and thoughts of self-harm, is nowadays considered one of the leading causes of maternal mortality in the first 12 months postpartum $(11,13-15)$. Furthermore, it has been documented that women reporting SI and thoughts during pregnancy or during the postpartum period had higher odds for developing postpartum depression $(16,17)$.

The increasing need of a careful assessment and screening of pregnant and nursing women's mental health should also take into account SI, thoughts of suicide, and thoughts about harming infants as well. Clinicians should carefully monitor and early identify related clinical manifestations, potential risk factors, and alarm symptoms related to suicide. Therefore, the present paper aims at providing a focused review about epidemiological data, risk and protective factors, and an overview about the main clinical correlates associated with the suicidal behavior during the pregnancy and postpartum period.

\section{MATERIALS AND METHODS}

The present review was carried out in accordance to the methods recommended by the Preferred Reporting Items for Systematic Reviews and Meta-Analyses (PRISMA) guidelines (18). Studies were identified searching the electronic databases MEDLINE, Embase, PsycINFO, and the Cochrane Library. A combined search strategy of free text terms and exploded MESH headings for the topics of suicide and perinatal period as following: ((suicide [Title/Abstract] OR suicide attempt [Title/ Abstract] OR suicidal ideation [Title/Abstract]) OR puerperium
[Title/Abstract]). ("suicide" [MeSH Terms] OR "suicide" [Title/ Abstract]) AND ("pregnancy" [MeSH Terms] OR "pregnancy" [Title/Abstract]) OR ("postpartum period” [MeSH Terms] OR "postpartum" [Title/Abstract] OR "postpartum period" [Title/ Abstract]) OR Studies published in English through March 20, 2015 were included. In addition, further studies were retrieved from reference listing of relevant articles and consultation with experts in the field and or manual search. Identified studies were independently reviewed for eligibility by two authors (Laura Orsolini and Alessandro Valchera) in a two-step based process; a first screening was performed based on title and abstract, while full texts were retrieved for the second screening. At both stages, disagreements by reviewers were resolved by consensus. Data were extracted by two authors (Laura Orsolini and Alessandro Valchera) and supervised by a third author (Cesario Bellantuono) using an ad hoc developed data extraction spreadsheet. With the initial set of keywords, 1,567 studies were identified. Of these, 219 were excluded because they were not in English, 1,219 were excluded because they were either duplicated or not consistent with the aims of the review. Of these remaining, 129 were relevant studies. Data were then analyzed according to the epidemiology, risk factors, and clinical correlates.

\section{RESULTS}

\section{Epidemiology}

A recent 15-year (1997-2012) retrospective study, from the UK National Confidential Inquiry that evaluated all suicides by people who had been in contact with psychiatric services, compared suicides among perinatal and non-perinatal women. Findings reported a suicide in perinatal period in $2 \%$ of women aged 16-50 years and in 4\% among women aged 20-35 years (19). A register-based cohort Danish study reported, among women with severe postpartum psychiatric disorders, a suicide risk drastically increased when compared with mother with no psychiatric history, by suggesting a strong correlation between perinatal suicidality and psychiatric conditions (20). A retrospective cohort study carried out on perinatal women at 24-28 weeks of gestation and 6 weeks postpartum reported an SI in $3.8 \%$ of the women screened with the Edinburgh Postnatal Depression Scale (EPDS), with only $1.1 \%$ with a high risk for suicide (i.e., with plan, intent, and access to suicidal means) (21). A register-based Swedish study reported a maternal suicide ratio of 3.7 per 100,000 live births for the period 1980-2007, being higher among women born in low-income countries (22). An audit cohort study evaluated 225 women afferent to a UK perinatal mental health team over a 12 -month period. Suicide attempts occurred among women with a previous postpartum depression in $24-49 \%$ (10). A prospective study evaluating thoughts of self-harm and SI during the postpartum period among women with mood disorders reported thoughts of self-harm and SI, respectively, in 16.97 and $6.16 \%$ of the sample, during the 1 -year postpartum period (23). A US community-based study reported a 14-day prevalence of antenatal SI of $2.7 \%$ (24). Findings from PND-ReScU reported a rate ranging $6.9-12 \%$ of suicidality during pregnancy, while a rate of $4.3-8.6 \%$ during the postpartum 
period, depending on the assessment tools (25). Women who had major or minor depressive episode during pregnancy showed a prevalence of suicidality of 26.4 and $34.1 \%$, while it was $18.4-30.6 \%$ during the postpartum period (25). A prospective cohort study evaluated the prevalence of SI as measured by the EPDS in a primary care population of women at 6-8 weeks postpartum screened for postnatal depressive symptoms. The authors reported that $4 \%$ of women in the community had SI occurring sometimes or quite often, while $9 \%$ reported any SI (26). Suicide attempts appear to be more frequent in the 1st and 12th months after delivery (27).

Furthermore, according to the NSW Australian Department of Health evaluating a 6-year time period, $73 \%$ of suicides by women within 1 year of birth were conducted by violent means (i.e., jumping from high place, lying in front of moving objects, gunshot, strangulation, and suffocation) $(14,22)$.

\section{Risk Factors}

Suicides in the perinatal period appear to be more likely occurring among women who are less likely to be receiving any active treatment at the time of death, younger maternal age, unpartnered relationship status, unplanned pregnancy, non-Caucasian race, with shorter illness duration, preexisting, and/or current psychiatric diagnosis $(9,19,21,24,26,28-34)$. Regarding the delivery, severe vaginal laceration was positively correlated with SI risk, while planned cesarean delivery was negatively associated (21). Furthermore, experiencing an intimate partner violence, including emotional abuse, physical, and/or sexual violence, seems to be more likely associated with suicidal thoughts during pregnancy and after childbirth (29, 35-37). A cross-sectional study evaluating a sample of pregnant teenagers found a significant association of suicidality with the 18- to 19-year-old subgroup, low education, prior abortion, physical abuse within the last 12 months, and current psychiatric disorders (38). Women who did not desire or had mixed feelings about being pregnant experienced a higher risk of SI (9). A case-control study comparing 520 women who were hospitalized for a postpartum suicide attempt with 2,204 control women who were not hospitalized for a postpartum suicide attempt concluded that maternal complications (i.e., labor, delivery complications, cesarean delivery, etc.) and adverse infant outcomes (i.e., preterm delivery, low birth weight, congenital malformations, etc.) were not associated with a hospitalization for a suicide attempt within 1 year after delivery (27). A cross-sectional analyses carried out on 234 pregnant women enrolled in a prospective cohort study in Brazil reported a higher likelihood of suicide risk among women with higher arachidonic acid and adrenic acid levels (39). Women who have had a postpartum psychiatric admission have a 70 times greater risk of suicide in their first postpartum year $(40,41)$.

All risk factors are summarized in Table $\mathbf{1 .}$

\section{Clinical Correlates}

Women who reported antenatal SI were more likely to experience comorbid antenatal MMD and antenatal panic disorder (24). In particular, preexisting and/or current psychiatric diagnoses
TABLE 1 | Risk factors for perinatal suicidality.

\begin{tabular}{|c|c|}
\hline \multicolumn{2}{|c|}{ Risk factors for perinatal suicidality } \\
\hline $\begin{array}{l}\text { Individual risk } \\
\text { factors }\end{array}$ & $\begin{array}{l}\text { - } \text { Younger age }(9,19,21,24,26,28-34) \\
\text { - } \text { Being unmarried }(9,19,21,24,26,28-34) \\
\text { - Personal and/or family history of psychiatric disorders } \\
\text { - } 9,19,21,24,25,28-34,42-46) \\
\text { - Personal and/or family history of suicidal attempt or } \\
\text { suicidal ideation }(9,19,21,24,25,28-34,42-46)\end{array}$ \\
\hline $\begin{array}{l}\text { Socioeconomical } \\
\text { risk factors }\end{array}$ & $\begin{array}{l}\text { - Family conflict (35-37) } \\
\text { - Exposure to (domestic) physical/psychological violence } \\
(35-37) \\
\text { - Loneliness and lack of social/family/partner support } \\
\text { (35-37) } \\
\text { - Partner who rejected paternity (35-37) }\end{array}$ \\
\hline $\begin{array}{l}\text { Environmental } \\
\text { risk factors }\end{array}$ & $\begin{array}{l}\text { - Social and gender inequalities }(28,37) \\
\text { - Social and racial discrimination }(28,37) \\
\text { - Belonging to an ethnic or religious minority }(28) \\
\text { - Crowded or inadequate housing }(24,28,37) \\
\text { - Living in rural areas }(37) \\
\text { - Exposure to disaster, conflict, war }(24)\end{array}$ \\
\hline $\begin{array}{l}\text { Gestational risk } \\
\text { factors }\end{array}$ & $\begin{array}{l}\text { - Unwanted/unintended pregnancy }(9,17) \\
\text { - Nulliparity }(32)\end{array}$ \\
\hline $\begin{array}{l}\text { Clinical risk } \\
\text { factors }\end{array}$ & $\begin{array}{l}\text { - Previous history of psychiatric disorders }(9,19,21,24 \text {, } \\
25,28-34,42-46) \\
\text { - Previous history of suicidal attempt or suicidal ideation } \\
(9,19,21,24,25,28-34,42-46) \\
\text { - Psychiatric comorbidity }(9,19,21,24,25,28-34, \\
42-46) \\
\text { - Shorter illness duration }(9,19,21,24,25,28-34,42-46) \\
\text { - Psychological symptoms (i.e., premenstrual irritability, } \\
\text { perceived pregnancy complications, negative attitude } \\
\text { toward the pregnancy, anxiety about birth, distancing } \\
\text { pattern of coping, etc.) }(9,21)\end{array}$ \\
\hline
\end{tabular}

represent strong risk factors, being MDD, comorbid anxiety disorders, sleep disturbances, or a substance and/or alcohol use disorder the most frequently identified $(9,19,21,24,25,28-34)$. A positive correlation with antenatal depression, lifetime bipolar disorder, and any current anxiety disorder as well as Beck Depression Inventory (BDI) scores $\geq 15$ and EPDS scores $\geq 11$ has been reported (42). Furthermore, poor subjective sleep quality was associated with increased odds of SI as well (43). Women with a dysphoric-dysregulated temperament are more likely to be at risk of suicide after delivering (44). Generally, an abrupt discontinuation of psychotropic medications during pregnancy has been linked to a higher risk of maternal suicidality (45). A history of a previous suicide attempts represents a strong risk for perinatal suicidality (46).

\section{DISCUSSION}

Although the incidence of suicide among women who have given birth during the past 12 months is lower than that of women who have not given birth, suicide still remains one of the most common leading causes of maternal death during the 1 year following delivery $(22,40,47)$. Perinatal suicide occurs mainly through more violent methods compared to suicide in non-pregnant women (11) and at a higher rate among women with a previous or current mental illness $(11,16)$. 
Despite very limited and contrasting studies, SI and suicides appear to be more likely to occur during pregnancy rather than postpartum (25).

However, the most important cognitive risk factor to consider in determining a risk of making a suicide attempt or dying by suicide is SI. SI predicts later suicidal behavior (including suicide and suicidal attempt) (48).

Therefore, a preventive and careful assessment, screening, and identification for SI should be included during the perinatal period (Table 2). However, a specific screening for suicide and SI is almost rare, mainly due to time constraints in prenatal care clinics, the lack of proper screening tools, and the missing collaboration between gynecologists/pediatricians and Mental Health's professionals (24). In fact, SI is usually assessed along with depression screening rather than with specifically designed tools (44, 49-51), such as the Scale for Suicide Ideation (SSRI), the Columbia-Suicide Severity Rating Scale (C-SSRS), and the Suicide Probability Scale (SPS) (2). Among the most widely used antepartum depression screening instruments, the Patient Health Questionnaire-9 (PHQ-9) (52) and the EPDS (51) represent easy tools that may be helpful both in primary care and community maternity services to screen perinatal depressive and anxiety disorders as well as SI $(53,54)$. PHQ-9 is a 9-item, depression screening scale (52). Item 9 ("thoughts that you would be better off dead, or of hurting yourself") of the PHQ-9, which assesses SI, has been correlated with item 10 of the EPDS (55). Subjects who answered "several days," "more than half the days," or "nearly every day" at item 9 were evaluated at risk of SI. While the EPDS (51), a 10-item self-report questionnaire, is usually administered to screen for postnatal depression. SI was defined as an answer of "sometimes" or "yes, quite often" to question 10 of the EPDS "The thought of harming myself has occurred to me."

\section{TABLE 2 | Risk assessment.}

Risk factors for perinatal suicidality

Clinical risk assessment $(9,19,21,24,25,28-34$, 42-46, 49-51)

SI risk assessment $(9,19,21$, 24, 25, 28-34, 42-46, 49-51)

Suicide plan risk assessment (44, 53-55)

Current or previous suicidal attempt risk assessment $(9,19,21,24,25,28-34$, 42-46, 49-51)

Estimating suicide risk (24, 44, 49-51)
- Current presentation of suicidality

- Psychiatric disorders

- History of current illness

- Current medications

- Psychosocial environment

- Current alcohol and/or drug use

- Individual strengths and vulnerabilities

- Nature

- Timing

- Persistence of the desire

- Intent of SI

- Lethality of the plan

- The level of detail and violence

- The level of access to means (e.g., weapon or store of medication)

- Timing

- Intent

- Method

- Consequences of the suicidal attempt

- Identification of protective and risk factors

- Determination of methods to mitigate/ strengthen these risk/protective risks
Further assessment tools may comprise the BDI at item 9 (50) and the Hamilton Rating Scale for Depression (HRSD) at item 3 $(49,56)$. The BDI is a 21 -item self-rated depression scale widely used to screen for depression. The HRSD is a clinician-rated scale. Girardi et al. (44) included the Suicidal History Self-rating Screening Scale in their perinatal assessment of women at risk of suicide.

Furthermore, women who are depressed and/or psychotic for suicide should be assessed for suicide as well. In presence of a SI or suicidal thought, clinicians should promptly developing a safety plan and referring to a specific psychiatric assessment as well as follow up the patient. In some case, hospitalization must be required as well. Urgency of referral depends on several factors including: whether SI is accompanied by a plan, whether there has been a history of suicide attempts, whether symptoms of a psychotic disorder are present. A risk assessment is helpful for identifying mothers at low-risk (SI or thought present, with a plan), medium-risk (SI with a plan or history of suicide attempt, without an immediate intent), or at high-risk (SI with an immediate intent). Warning signs of the risk of imminent suicide may include "feeling trapped," "worthless, hopelessness, talking about death, writing a will, hoarding medication," etc.

Suicide ideation is more likely associated with unplanned pregnancies, current mood and/or comorbid anxiety disorders, previous SI and/or suicidal attempt, and younger maternal age $(9,19,21,24,26,28-34)$.

Generally, screening during the perinatal period (particularly during pregnancy) represents an essential clinical tool for identifying women at higher risk of perinatal suicidality. Longterm identification and support of women at particular risk of maternal death due to suicide in the first year following birth may help lower the incidence of late maternal deaths. Overall, it should be proposed to clinicians, particularly gynecologists and primary care physicians, to ask to all pregnant women about their personal mental health history and family history. Women with a previous history of mental disorder (particularly, bipolar and MDDs as well as psychoses) should be offered a mental health assessment antenatally and managed by a psychiatrist. In addition, a regular interview on lifetime SI should be performed. Furthermore, mothers should be regularly monitored and supported for at least 12 months following delivery.

\section{AUTHOR CONTRIBUTIONS}

$\mathrm{LO}, \mathrm{AV}$, and $\mathrm{CB}$ conceived the topic of the manuscript, while LO, $\mathrm{RV}$, and MF carried out the main analysis. CT and FI assisted in either screening of the studies or preparation of the attachments. DB, MP, and GP served as study reviewers. CB and GP served as senior study reviewers. All the coauthors substantially contributed to the present piece of work before approving it for final submission.

\section{FUNDING}

There is no funding source to disclose in conjunction with the present piece of work. 


\section{REFERENCES}

1. Mathers CD, Loncar D. Projections of global mortality and burden of disease from 2002 to 2030. PLoS Med (2006) 3(11):e.442. doi:10.1371/journal. pmed.0030442

2. Koslow SH, Ruiz P, Nemeroff CB. A Concise Guide to Understanding Suicide. Cambridge: Cambrdige University Press (2014).

3. Oquendo MA, Halberstam B, Mann JJ. Risk factors for suicidal behavior: utility and limitations of research instruments. In: First MB, editor. Standardized Evaluation in Clinical Practice (2003). p. 103-30.

4. Borges G, Nock MK, Haro Abad JM, Hwang I, Sampson NA, Alonso J, et al. Twelve month prevalence of and risk factors for suicide attempts in the WHO World Mental Health Surveys. J Clin Psychiatry (2010) 71(12):1617. doi:10.4088/JCP.08m04967blu

5. Brown GK, Currier G, Stanley B. Suicide attempt registry pilot project. National Institute of Mental Health Annual Meeting of the Developing Centers for Intervention and Prevention of Suicide, September 2008. Canandaigua, NY (2008).

6. MöllerHJ. Suicide, suicidality and suicide prevention in affective disorders. Acta Psychiatr Scand Suppl (2003) 108(418):73-80. doi:10.1034/j.1600-0447.108. s418.15.x

7. Posner K, Oquendo MA, Gould M, Stanley B, Davies M. Columbia Classification Algorithm of Suicide Assessment (C-CASA): classification of suicidal events in the FDA's pediatric suicidal risk analysis of antidepressants. Am J Psychiatry (2007) 164(7):1035-43. doi:10.1176/ajp.2007.164.7.1035

8. Jones I, Chandra PS, Dazzan P, Howard LM. Bipolar disorder, affective psychosis, and schizophrenia in pregnancy and the post-partum period. Lancet (2014) 384(9956):1789-99. doi:10.1016/S0140-6736(14)61278-2

9. Newport DJ, Levey LC, Pennell PB, Ragan K, Stowe ZN. Suicidal ideation in pregnancy: assessment and clinical implications. Arch Womens Ment Health (2007) 10(5):181-7. doi:10.1007/s00737-007-0192-x

10. Healey C, Morriss R, Henshaw C, Wadoo O, Sajjad A, Scholefield H, et al. Self-harm in postpartum depression and referrals to a perinatal mental health team: an audit study. Arch Womens Ment Health (2013) 16(3):237-45. doi:10.1007/s00737-013-0335-1

11. Lindahl V, Pearson JL, Colpe L. Prevalence of suicidality during pregnancy and the postpartum. Arch Womens Ment Health (2005) 8(2):77-87. doi:10.1007/ s00737-005-0080-1

12. Copersino ML, Jones H, Tuten M, Svikis D. Suicidal ideation among drug-dependent treatment-seeking inner-city pregnant women. J Maint Addict (2008) 3(2-4):53-64. doi:10.1300/J126v03n02_07

13. Palladino CL, Singh V, Campbell J, Flynn H, Gold KJ. Homicide and suicide during the perinatal period: findings from the National Violent Death Reporting System. Obstet Gynecol (2011) 118(5):1056-63. doi:10.1097/ AOG.0b013e31823294da

14. Thornton C, Schmied V, Dennis CL, Barnett B, Dahlen HG. Maternal deaths in NSW (2000-2006) from nonmedical causes (suicide and trauma) in the first year following birth. Biomed Res Int (2013) 2013:623743. doi:10.1155/2013/623743

15. Fuhr DC, Calvert C, Ronsmans C, Chandra PS, Sikander S, De Silva MJ, et al. Contribution of suicide and injuries to pregnancy-related mortality in low-income and middle-income countries: a systematic review and meta-analysis. Lancet Psychiatry (2014) 1(3):213-25. doi:10.1016/S2215-0366(14) 70282-2

16. Do T, Hu Z, Otto J, Rohrbeck P. Depression and suicidality during the postpartum period after first time deliveries, active component service women and dependent spouses, U.S. Armed Forces, 2007-2012. MSMR (2013) 20(9):2-7.

17. Turkcapar AF, Kadığlu N, Aslan E, Tunc S, Zayıfoğlu M, Mollamahmutoğlu L. Sociodemographic and clinical features of postpartum depression among Turkish women: a prospective study. BMC Pregnancy Childbirth (2015) 3(15):108. doi:10.1186/s12884-015-0532-1

18. Liberati A, Altman DG, Tetzlaff J, Mulrow C, Gøtzsche PC, Ioannidis JP, et al. The PRISMA statement for reporting systematic reviews and meta-analyses of studies that evaluate health care interventions: explanation and elaboration. Ann Intern Med (2009) 151(4):W65-94. doi:10.7326/0003-4819-151-4200908180-00136

19. Khalifeh H, Hunt IM, Appleby L, Howard LM. Suicide in perinatal and non-perinatal women in contact with psychiatric services: 15 year findings from a UK national inquiry. Lancet Psychiatry (2016) 3(3):233-42. doi:10.1016/ S2215-0366(16)00003-1

20. Johannsen BM, Larsen JT, Laursen TM, Bergink V, Meltzer-Brody S, Munk-Olsen T. All-cause mortality in women with severe postpartum psychiatric disorders. Am J Psychiatry (2016) 173(6):635-42. doi:10.1176/appi. ajp.2015.14121510

21. Celik C, Ozdemir B, Oznur T. Suicide risk among perinatal women who report thoughts of self-harm on depression screens. Obstet Gynecol (2015) 126(1):216-7. doi:10.1097/AOG.0000000000000941

22. Esscher A, Essén B, Innala E, Papadopoulos FC, Skalidou A, SundströmPoromaa I, et al. Suicides during pregnancy and 1 year postpartum in Sweden, 1980-2007. Br J Psychiatry (2016) 208(5):462-9. doi:10.1192/bjp. bp.114.161711

23. Pope CJ, Xie B, Sharma V, Campbell MK. A prospective study of thoughts of self-harm and suicidal ideation during the postpartum period in women with mood disorders. Arch Womens Ment Health (2013) 16(6):483-8. doi:10.1007/ s00737-013-0370-y

24. Gavin AR, Tabb KM, Melville JL, Guo Y, Katon W. Prevalence and correlates of suicidal ideation during pregnancy. Arch Womens Ment Health (2011) 14(3):239-46. doi:10.1007/s00737-011-0207-5

25. Mauri M, Oppo A, Borri C, Banti S; PND-ReScU group. SUICIDALITY in the perinatal period: comparison of two self-report instruments. Results from PND-ReScU. Arch Womens Ment Health (2012) 15(1):39-47. doi:10.1007/ s00737-011-0246-y

26. Howard LM, Flach C, Mehay A, Sharp D, Tylee A. The prevalence of suicidal ideation identified by the Edinburgh Postnatal Depression Scale in postpartum women in primary care: findings from the RESPOND trial. BMC Pregnancy Childbirth (2011) 3(11):57. doi:10.1186/1471-2393-11-57

27. Schiff MA, Grossman DC. Adverse perinatal outcomes and risk for postpartum suicide attempt in Washington state, 1987-2001. Pediatrics (2006) 118(3):e669-75. doi:10.1542/peds.2006-0116

28. Kim JJ, La Porte LM, Saleh MP, Allweiss S, Adams MG, Zhou Y, et al. Suicide risk among perinatal women who report thoughts of self-harm on depression screens. Obstet Gynecol (2015) 125(4):885-93. doi:10.1097/ AOG.0000000000000718

29. Sit D, Luther J, Buysse D, Dills JL, Eng H, Okun M, et al. Suicidal ideation in depressed postpartum women: associations with childhood trauma, sleep disturbance and anxiety. J Psychiatr Res (2015) 6(6-67):95-104. doi:10.1016/j. jpsychires.2015.04.021

30. Huang H, Faisal-Cury A, Chan YF, Tabb K, Katon W, Menezes PR. Suicidal ideation during pregnancy: prevalence and associated factors among low-income women in São Paulo, Brazil. Arch Womens Ment Health (2012) 15(2):135-8. doi:10.1007/s00737-012-0263-5

31. Tavares D, Quevedo L, Jansen K, Souza L, Pinheiro R, Silva R. Prevalence of suicide risk and comorbidities in postpartum women in Pelotas. Rev Bras Psiquiatr (2012) 34(3):270-6. doi:10.1016/j.rbp.2011.12.001

32. Farias DR, Pinto Tde J, Teofilo MM, Vilela AA, Vaz Jdos S, Nardi AE, et al. Prevalence of psychiatric disorders in the first trimester of pregnancy and factors associated with current suicide risk. Psychiatry Res (2013) 210(3):962-8. doi:10.1016/j.psychres.2013.08.053

33. Chen YH, Lau G. Maternal deaths from suicide in Singapore. Singapore Med $J(2008)$ 49(9):694-7.

34. Comtois KA, Schiff MA, Grossman DC. Psychiatric risk factors associated with postpartum suicide attempt in Washington State, 1992-2001. Am J Obstet Gynecol (2008) 199:120.e1-5. doi:10.1016/j.ajog.2008.02.011

35. Alhusen JL, Frohman N, Purcell G. Intimate partner violence and suicidal ideation in pregnant women. Arch Womens Ment Health (2015) 18(4):573-8. doi:10.1007/s00737-015-0515-2

36. Stewart DE. The importance of intimate partner violence and suicidal ideation in pregnant women. Arch Womens Ment Health (2015) 18(4):571-2. doi:10.1007/s00737-015-0539-7

37. Fisher J, Tran TD, Biggs B, Dang TH, Nguyen TT, Tran T. Intimate partner violence and perinatal common mental disorders among women in rural Vietnam. Int Health (2013) 5(1):29-37. doi:10.1093/inthealth/ihs012

38. Coelho FM, Pinheiro RT, Silva RA, de Ávila Quevedo L, de Mattos Souza LD, de Matos MB, et al. Parental bonding and suicidality in pregnant teenagers: a population-based study in southern Brazil. Soc Psychiatry Psychiatr Epidemiol (2014) 49(8):1241-8. doi:10.1007/s00127-014-0832-1 
39. Vaz JS, Kac G, Nardi AE, Hibbeln JR. Omega-6 fatty acids and greater likelihood of suicide risk and major depression in early pregnancy. J Affect Disord (2014) 15(2-154):76-82. doi:10.1016/j.jad.2013.04.045

40. Appleby L, Mortensen PB, Faragher EB. Suicide and other causes of mortality after post-partum psychiatric admission. Br J Psychiatry (1998) 173:209-11. doi:10.1192/bjp.173.3.209

41. Oates M. Perinatal psychiatric disorders: a leading cause of maternal morbidity and mortality. Br Med Bull (2003) 67:219-29. doi:10.1093/bmb/ldg011

42. Castro e Couto T, Brancaglion MY, Cardoso MN, Faria GC, Garcia FD, Nicolato R, et al. Suicidality among pregnant women in Brazil: prevalence and risk factors. Arch Womens Ment Health (2016) 19(2):343-8. doi:10.1007/ s00737-015-0552-x

43. Gelaye B, Barrios YV, Zhong QY, Rondon MB, Borba CP, Sánchez SE, et al. Association of poor subjective sleep quality with suicidal ideation among pregnant Peruvian women. Gen Hosp Psychiatry (2015) 37(5):441-7. doi:10.1016/ j.genhosppsych.2015.04.014

44. Girardi P, Pompili M, Innamorati M, Serafini G, Berrettoni C, Angeletti G, et al. Temperament post-partum depression, hopelessness, and suicide risk among women soon after delivering. Women Health (2011) 51(5):511-24. do i: $10.1080 / 03630242.2011 .583980$

45. Einarson A, Selby P, Koren G. Abrupt discontinuation of psychotropic drugs during pregnancy: fear of teratogenic risk and impact of counselling. J Psychiatry Neurosci (2001) 26(1):44-8.

46. Pinheiro RT, de Silva RA, Magalhães PV, Horta BL, Pinheiro KA. Two studies on suicidality in the postpartum. Acta Psychiatr Scand (2008) 118(2):160-3. doi:10.1111/j.1600-0447.2008.01184.x

47. Turner LA, Kramer MS, Liu S. Maternal mortality and morbidity study group of the Canadian perinatal surveillance system. Cause-specific mortality during and after pregnancy and the definition of maternal death. Chronic Dis Can (2002) 23(1):31-6.

48. Mundt JC, Greist JH, Jefferson JW, Federico M, Mann JJ, Posner K. Prediction of suicidal behaviour in clinical research by lifetime suicidal ideation and behavior ascertained by the electronic Columbia-Suicide Severity Rating Scale. J Clin Psychiatry (2013) 74(9):887-93. doi:10.4088/JCP.13m08398

49. Hamilton M. A rating scale for depression. JNeurol Neurosurg Psychiatry (1960) 23:56-62. doi:10.1136/jnnp.23.1.56
50. Beck AT, Ward CH, Mendelson M, Mock J, Erbaugh J. An inventory for measuring depression. Arch Gen Psychiatry (1961) 4:561-71. doi:10.1001/ archpsyc.1961.01710120031004

51. Cox JL, Holden JM, Sagovsky R. Detection of postnatal depression. Development of the 10-item Edinburgh Postnatal Depression Scale. Br J Psychiatry (1987) 150:782-6. doi:10.1192/bjp.150.6.782

52. Kroenke K, Spitzer RL, Williams JB. The PHQ-9: validity of a brief depression severity measure. J Gen Intern Med (2001) 16(9):606-13. doi:10.1046/ j.1525-1497.2001.016009606.x

53. Gibson J, McKenzie-McHarg K, Shakespeare J, Price J, Gray R. A systematic review of studies validating the Edinburgh Postnatal Depression Scale in antepartum and postpartum women. Acta Psychiatr Scand (2009) 119(5):350-64. doi:10.1111/j.1600-0447.2009.01363.x

54. Matthey S. Using the Edinburgh Postnatal Depression Scale to screen for anxiety disorders. Depress Anxiety (2008) 25(11):926-31. doi:10.1002/da.20415

55. Zhong QY, Gelaye B, Rondon MB, Sánchez SE, Simon GE, Henderson DC, et al. Using the Patient Health Questionnaire (PHQ-9) and the Edinburgh Postnatal Depression Scale (EPDS) to assess suicidal ideation among pregnant women in Lima, Peru. Arch Womens Ment Health (2015) 18(6):783-92. doi:10.1007/s00737-014-0481-0

56. Williams JA. A structured interview guide for the Hamilton Depression Rating Scale. Arch Gen Psychiatry (1988) 45:742-7. doi:10.1001/archpsyc. 1988.01800320058007

Conflict of Interest Statement: The authors declare that the research was conducted in the absence of any commercial or financial relationships that could be construed as a potential conflict of interest.

Copyright (C) 2016 Orsolini, Valchera, Vecchiotti, Tomasetti, Iasevoli, Fornaro, De Berardis, Perna, Pompili and Bellantuono. This is an open-access article distributed under the terms of the Creative Commons Attribution License (CC BY). The use, distribution or reproduction in other forums is permitted, provided the original author(s) or licensor are credited and that the original publication in this journal is cited, in accordance with accepted academic practice. No use, distribution or reproduction is permitted which does not comply with these terms. 